\title{
DEPRESSION TREATMENT PREFERENCES IN HOME HEALTHCARE
}

\author{
Patrick J. Raue, Ph.D., Mark I. Weinberger, Ph.D., MPH, Jo Anne Sirey, Ph.D., Barnett S. \\ Meyers, M.D., and Martha L. Bruce, Ph.D., M.P.H.
}

\section{Abstract}

Objective-The authors hypothesized that the depression treatment preferences of elderly home care patients would vary by depression experience, with preferences for active treatments being associated with current depression and antidepressant treatment.

Methods-The authors conducted cross sectional secondary analyses of the TRIAD ("Training in the Assessment of Depression") study of 256 randomly selected elderly patients newly admitted to homecare. The study assessed depression treatment preference for active (i.e., medication or psychotherapy) versus non-active or complementary approaches (e.g., religious activities, do nothing). Two separate indicators of depression experience were used: diagnoses of major or minor depression, and current or previous antidepressant treatment.

Results-121 patients (48\%) preferred active treatment with either medication or psychotherapy as their first choice, while the remainder preferred non-active or complementary approaches. Logistic regression indicated that current antidepressant use, previous psychotherapy experience, white and Hispanic versus African-American patients, greater IADL impairment, and less personal stigma about depression were independently associated with preference for an active treatment.

Conclusions-Elderly homecare patients have a variety of depression treatment preferences ranging from active treatments, to religious or spiritual activities, to no treatment. Several factors were associated with a preference for active treatment, including treatment experience, co-existing physical impairment, culture, and attitudes and beliefs. Understanding patient preferences may form the basis of engaging older depressed home care patients in treatment.

\section{Keywords}

Depression treatment preferences; home health care; geriatrics

\begin{abstract}
Major depressive disorder and milder forms of depression are disproportionately high among home healthcare patients, approaching $14 \%$ and $11 \%$ respectively (1). While effective pharmacological (2) and psychological treatments $(3,4)$ exist for depression in older adults, depression remains under-treated in the homecare setting $(1,5,6)$ for a variety of reasons including patient reluctance to initiate and adhere to recommended treatments.

Examination of patient preferences for depression treatment may shed light on this reluctance, and may identify subgroups of patients more likely to prefer such treatment. The majority of research on treatment preferences has been conducted in primary care settings (7-16), with little known about the preferred mental health treatment approaches of elderly homecare patients. A pilot study found that prayer was the most preferred approach
\end{abstract}


identified by elderly long-term care patients (17). Personal experience with depression, however, increased patient preference for medication or psychotherapy (17).

Using cross sectional data from the TRIAD ("Training in the Assessment of Depression") study of elderly patients newly admitted to homecare $(18,19)$, we examined the treatment preferences of this understudied population at high risk for experiencing depression. Previous work suggests that depression experience, namely diagnoses of major or minor depression or use of antidepressant medication $(7,12,14,17)$, would be associated with preferences for an active treatment such as antidepressant medication or psychotherapy. We also explored the association of other sociodemographic and clinical variables with preferences for an active treatment.

\section{METHODS}

Overview

The authors conducted a secondary analysis of data collected as part of TRIAD ("Training in the Assessment of Depression"), a nurse-randomized trial of randomly selected patients $(18,19)$. TRIAD was conducted in three Medicare-certified home healthcare agencies (CHHAs) serving patients throughout Westchester County and neighboring locations in New York State. The study was approved by the Weill Cornell Medical College IRB. All patients provided written informed consent. Recruitment began 5/2004 and follow-up interviews ended 6/2005.

\section{Study Participations}

Patient eligibility criteria included: age $\geq 65$ years, English speaking, nonaphasic, not hearing impaired, and nondemented according to agency start of care records; and able to give consent. An additional eligibility criteria was Mini-Mental State Exam (MMSE) (20) scores $\geq 20$, as assessed by study research assistants (see below). Typical of acute home healthcare nationwide, no patient had been referred to homecare services following discharge from an inpatient psychiatric facility. A designated agency staff person selected the first consecutively admitted eligible patient per week, and telephoned the patient for permission to be contacted by research staff for possible study participation. Researchers contacted 477 patients, of whom 256 (54\%) enrolled, 84 (18\%) were not eligible, and 120 $(25 \%)$ refused. Patients were interviewed in their home (see Bruce et al. (18) for a full description of the study design). Analyses for the current analyses focused on the baseline interview.

\section{Patient Measures}

Depression symptoms were assessed by research assistants trained in administering the Structured Clinical Interview for Axis I DSM-IV Disorders (SCID) (21), with diagnoses of major and minor depression assigned by the study psychiatrist and psychologist after review of symptoms. Current and previous antidepressant use was determined by review of patients' medications and by self-report. Although we were unable to determine whether low dose antidepressants had been prescribed for purposes other than treating depression, such as for pain management or sleep disturbances, we took the conservative approach of including these approved antidepressants. We created a 3-level variable reflecting current antidepressant use, previous but not current use, and no history of use.

Depression treatment preferences were assessed with the Cornell Treatment Preference Index (15), a modified version of a measure used in other primary care studies (22). This measure consists of the question: "Based on your experience and how you feel right now, which of the following treatments would be your $1^{\text {st }}$ choice, $2^{\text {nd }}$, and $3^{\text {rd }}$ choice?" 
Participants were asked to select from the following treatment preferences: antidepressant treatment, individual psychotherapy, group psychotherapy, combined antidepressant medication and psychotherapy, herbal remedies, religious or spiritual activities, exercise, other activity, or "do nothing." If patients were not currently depressed they were asked to select treatments "if you were suffering from a serious depression."

The 24-item Hamilton Depression Rating Scale (HDRS) (23) measured depression severity for all participants. Functional status was measured using counts of disability in basic (ADL) and instrumental (IADL) activities of daily living (24). Medical morbidity was estimated using the Chronic Disease Score, a measure constructed from medications updated to the 2005 American Hospital Formulary (25). Cognitive impairment was assessed with the MiniMental State Exam (MMSE) (20). Anxiety was assessed with the Clinical Anxiety Scale (26). "Public stigma" was defined using the mean of 3 items reflecting beliefs regarding society's attitudes about depressed individuals ("Most people would willingly accept a person who has had depression as a close friend") (27). This index was derived from the 12item stigma scale (27) shown to predict treatment discontinuation among older depressed adults (28). "Personal stigma" translated the index questions from "most people" to "I" (e.g., "I would willingly accept a person who has had depression as a close friend").

\section{Statistical Analysis}

Univariate analyses with mean and standard deviation for continuous variables and frequency and percentage for categorical variables are used to describe the sample. We report the percentage of patients who chose each depression treatment option as their first choice. We coded these choices into active (i.e., medication or psychotherapy) versus nonactive or complementary approaches (e.g., religious activities, do nothing). We used chi square to test the association of diagnoses of major or minor depression with patient preference for an active treatment with either medication or psychotherapy. We also used chi square to test the association of our 3-level variable reflecting current antidepressant use, previous but not current use, and no history of use, with preference for active treatment. We then conducted bivariate analyses to identify associations among other sociodemographic and clinical variables and preference for active treatment, using chi-square for categorical variables and t-test for continuous variables. Variables significant at the 10 level were entered into a multivariate logistic regression to determine independent predictors. History of antidepressant use (current, past, never) was represented in the regression models by dummy variables. The final model included only those variables that were significant at the . 05 level.

Analyses were conducted using SPSS, version 14.0 (29). A two-tailed alpha level of .05 was used for each statistical test.

\section{RESULTS}

Patient age ranged from 65 to 96 years (mean=78.2 \pm 7.1 ), $62 \%$ were female, and $16 \%$ classified themselves as ethnic/racial minorities. On average, clinical and functional measures indicated substantial medical morbidity and disability, with the Chronic Disease Scale averaging 6.2 and reported limitations in activities of daily living averaging 1.4 (ADL) and 3.5 (IADL). $7 \%$ of patients (19/256) met criteria for major depression as assessed by the SCID and $9 \%$ for minor depression (22/256). 20\% of all patients (51/256) were currently taking an antidepressant. $26 \%$ of patients with major depression (5/19), $36 \%$ with minor depression (8/22), and $18 \%$ with no depression diagnosis (38/215) were taking an antidepressant. $8 \%(21 / 256)$ reported previous but not current antidepressant use. No patient reported receiving psychotherapy upon admission to homecare, while 65 (25\%) reported previous participation in psychotherapy. 
Figure 1 presents patients' reported first choice treatment options. 47\% of patients (121/256) preferred an active treatment as their first choice (e.g., antidepressant medication, psychotherapy, or their combination), while the remaining 53\% preferred an inactive or complementary treatment approach (e.g., religious or spiritual activities, exercise). $77 \%$ of those with current antidepressant use preferred an active treatment, in comparison to $57 \%$ of those with past use and $37 \%$ of those with no history of use (chi square $=25.69, \mathrm{df}=2, \mathrm{p}<$. 001). Current diagnosis of major or minor depression was unrelated to such preference.

Other sociodemographic and clinical variables associated with preference for active treatment at the .10 significance level were male gender, higher education, previous psychotherapy, race (white or Hispanic versus Black), greater IADL impairment, and less personal stigma (Table 1). The final logistic regression model indicated that current antidepressant use, previous psychotherapy, white or Hispanic race, greater IADL impairment, and less personal stigma were independently associated with preference for an active treatment (Table 2). Restricting the sample to only those subjects who were not currently taking an antidepressant yielded similar findings (Table 2).

\section{DISCUSSION}

Elderly homecare patients express a variety of depression treatment preferences ranging from active treatments, to religious or spiritual activities, to "doing nothing." Relatively equivalent numbers of patients preferred antidepressant medication (19\%) in comparison to psychotherapy (18\%). Fewer patients preferred combined treatment $(7 \%)$ or group psychotherapy (4\%). The most commonly preferred non-active or complementary approaches were religious or spiritual activities (15\%) and exercise (13\%). A number of patients preferred to "do nothing" in response to depression (10\%).

Among patients who were currently depressed, only 26\% (13/51) were currently taking an antidepressant and none were receiving psychotherapy. We may speculate that many of these patients were not receiving treatment either because they did not want to be treated, or because no effort had been made to engage them in treatment decisions about depression care. In addition, a sizable number of patients who were not currently depressed were taking an antidepressant $(18 \% ; 38 / 215)$. This may reflect appropriate maintenance treatment for depression, or antidepressant prescription for reasons other than depression.

Our hypothesis that preferences for an active treatment with either medication or psychotherapy would be related to "depression experience" was partially confirmed. Patients who were currently taking an antidepressant medication, or who had taken one in the past, were more likely to prefer an active treatment in comparison to patients who had never taken an antidepressant. Patients currently taking an antidepressant had nearly a four-fold increase in preferring an active treatment compared with patients not on antidepressants. Previous experience with psychotherapy was also independently associated with preference for an active treatment. These findings are consistent with those in primary care $(7,12,14)$ and long-term homecare patients (17). They suggest that the more experience patients have with treatment for depression, the more comfort or interest they have in pursuing (or continuing to pursue) such treatment.

Contrary to our hypothesis, current diagnosis of major or minor depression was not associated with patient preference for an active treatment, nor was our measure of depression severity. We predicted that the more individuals personally felt the distress of depression, the more they would prefer an active treatment approach, particularly in comparison to non-depressed patients for whom the hypothetical clinical scenario regarding depression treatment would be less salient. Our ratings of symptoms, however, may not have 
captured perceived distress, which has been shown to be a better predictor of treatment initiation and adherence $(30,31)$.

Logistic regression revealed that white or Hispanic versus Black patients, those with greater IADL impairment, and those with less personal stigma were also more likely to prefer an active treatment. Restricting the sample to those subjects who were not currently taking an antidepressant yielded similar findings. The finding on race is consistent with previous research showing that Black individuals have negative attitudes and beliefs about antidepressant medication in particular $(7,10,32)$, and are less likely to adhere to depression treatment than white patients $(33,34)$. The finding on IADL impairment may indicate a greater experience with, and consequent acceptance of standard medical treatments.

However, neither medical burden nor number of medications was associated with preference for active depression treatment. Perhaps IADL impairment is a better indicator of distress or impairment associated with depression than other medical variables. Alternatively, those who acknowledge a need for help, as exemplified by reports of IADL limitations, may be more likely to recognize the value of active depression treatment.

Stigmatic concerns and negative beliefs about depression and mental health treatment may influence the perception of such treatment as unacceptable, and may lead to preferences for non-active or complementary approaches (35) and to poor medication adherence $(28,30)$. Personal stigma (an individual's attitudes about depressed individuals) was associated with a preference for active treatment, whereas public stigma (an individual's beliefs about society's attitudes) was not. Data from older adults receiving home delivered meals indicate that personal stigma may be particularly meaningful for individuals whose functional disabilities result in a smaller social sphere and less concern with society's attitudes (36). Also consistent with this finding, "self-stigma" but not public stigma was associated with attitudes about mental health treatment among diverse community-dwelling older adults (32). The socio-demographic determinants of stigma in older adults, and their impact on the treatment engagement process, are promising areas for future investigation.

Limitations of the study include assessment of treatment preferences using hypothetical questions for patients who were not currently depressed, if they were "suffering from a serious depression." We do not know whether patients who were depressed considered themselves to be suffering from a "serious" depression, and whether this belief would be related to treatment preferences. In addition, actual treatment decisions, initiation, and adherence may differ when patients are presented with real-life treatment choices.

Moreover, opportunities for home healthcare patients to engage in treatment consistent with their preferences may be constrained by practical issues such as the availability of different treatment approaches in this setting. Another study limitation is that the sample had relatively low levels of depression compared to other studies in homecare (1), in part given that data were collected for purposes of an intervention as opposed to a prevalence study. Our sample from 2004 also had low levels of antidepressant use (20\%) relative to more recent 2007 home healthcare data (34\%) (37). Our rate however is higher than 5 years prior $(11 \%)(1)$, reflecting an increasing trend over time. Thus, the distribution of patient preferences may not be generalizable to the current home healthcare population. Findings on variables associated with treatment preferences, however, should not be affected by the above potential sample biases. Another study limitation is that we did not have power to separately examine factors associated with a preference for antidepressant medications and for psychotherapy. Findings relate to preferences for active treatments in general. Lastly, we do not have data on past history of depressive disorders, and so findings concern the impact of current depression and previous treatment experience. 


\section{CONCLUSIONS}

Elderly homecare patients express a wide variety of depression treatment preferences, from active treatments, to religious or spiritual activities, to "doing nothing." Home healthcare services represent a unique opportunity to engage the homebound population, characterized by high levels of medical morbidity and disability, in appropriate depression treatment. Our findings on variables associated with such preferences shed light on the reluctance of subgroups of homecare patients to engage in active treatment. Given the positive impact of meeting patients' depression treatment preferences in primary care settings (15), and the potential value of shared decision-making interventions $(38,39,40)$, such interventions may also be fruitfully investigated in the homecare setting.

\section{Acknowledgments}

Disclosures: Dr. Bruce has research support from NIH and consulting relationships with NIH projects funded to MediSpin, Inc. Dr. Meyers has received research support from Forest Laboratories. Dr. Meyeres has received honoraria from and has been a consultant to Corcept, Forest Laboratories, Pfizer Inc. and Organon.

Grant support: National Institute of Mental Health R24 MH64608; K23 MH069784; R01 MH084872; R01 MH082425

The authors thank the nurses, other clinicians, administrators, and support staff at their partnering agencies: Dominican Sisters Family Health Services, Visiting Nurse Association of Hudson Valley, and Visiting Nurse Services in Westchester.

\section{REFERENCES}

1. Bruce ML, McAvay GJ, Raue PJ, et al. Major depression in elderly home health care patients. American Journal of Psychiatry. 2002; 159:1367-1374. [PubMed: 12153830]

2. Charney DS, Reynolds CF 3rd, Lewis L, et al. Depression and Bipolar Support Alliance consensus statement on the unmet needs in diagnosis and treatment of mood disorders in late life. Archives of General Psychiatry. 2003; 60:664-672. [PubMed: 12860770]

3. Arean PA, Cook B. Psychotherapy and combined psychotherapy/ pharmacotherapy for late life depression. Biological Psychiatry. 2002; 52:293-303. [PubMed: 12182934]

4. Engels GI, Vermey M. Efficacy of non-medical treatments of depression in elders: a quantitative analysis. Journal of Clinical Geropsychology. 1997; 3:17-35.

5. Brown EL, McAvay G, Raue PJ, et al. Recognition of depression among elderly recipients of home care services. Psychiatric Services. 2003; 54:208-213. [PubMed: 12556602]

6. Brown EL, Bruce ML, McAvay GJ, et al. Recognition of late-life depression in home care: accuracy of the outcome and assessment information set. Journal of the American Geriatric Society. 2004; 52:995-999.

7. Dwight-Johnson M, Sherbourne C, Liao D, et al. Treatment preferences among depressed primary care patients. Journal of General Internal Medicine. 2000; 15:527-534. [PubMed: 10940143]

8. Dwight-Johnson M, Lagomasino IT, Aisenberg E, et al. Using conjoint analysis to assess depression treatment preferences among low-income Latinos. Psychiatric Services. 2004; 55:934-936. [PubMed: 15292545]

9. Churchill R, Khaira M, Gretton V, et al. Treating depression in general practice: factors affecting patients' treatment preferences. The British Journal of General Practice. 2000; 50:905-906. [PubMed: 11141877]

10. Cooper LA, Gonzales JJ, Gallo JJ, et al. The acceptability of treatment for depression among African-American, Hispanic, and white primary care patients. Medical Care. 2003; 41:479-489. [PubMed: 12665712]

11. Cooper-Patrick L, Powe N, Jenckes M, et al. Identification of patient attitudes and preferences regarding treatment of depression. Journal of General Internal Medicine. 1997; 12:431-438. [PubMed: 9229282] 
12. Gum AM, Arean PA, Hunkeler E, et al. Depression treatment preferences in older primary care patients. The Gerontologist. 2006; 46:14-22. [PubMed: 16452280]

13. Landreville P, Landry J, Baillargeon L, et al. Older adults' acceptance of psychological and pharmacological treatments for depression. The Journals of Gerontology Series B: Psychological Sciences and Social Sciences. 2001; 56:285-291.

14. Lin P, Campbell DG, Chaney EF, et al. The influence of patient preference on depression treatment in primary care. Annals of Behavioral Medicine. 2005; 30:164-173. [PubMed: 16173913]

15. Raue PJ, Schulberg HC, Heo M, et al. Patients' depression treatment preferences and initiation, adherence, and outcome: a randomized primary care study. Psychiatric Services. 2009; 60:337343. [PubMed: 19252046]

16. van Schaik DJ, Klijn AF, van Hout HP, et al. Patients' preferences in the treatment of depressive disorder in primary care. General Hospital Psychiatry. 2004; 26:184-189. [PubMed: 15121346]

17. Fyffe DC, Brown EL, Sirey JA, et al. Older home-care patients' preferred approaches to depression care: a pilot study. Journal of Gerontological Nursing. 2008; 34:17-22. [PubMed: 18714602]

18. Bruce ML, Brown EL, Raue PJ, et al. A randomized trial of depression assessment intervention in home health care. Journal of the American Geriatric Society. 2007; 55:1793-1800.

19. Brown EL, Raue PJ, Roos BA, et al. Training nursing staff to recognize depression in home healthcare. Journal of the American Geriatric Society. 2010; 58:122-128.

20. Folstein MF, Folstein SE, McHugh PR. Mini-mental state. A practical method for grading the cognitive state of patients for the clinician. Journal of Psychiatric Research. 1975; 12:189-198. [PubMed: 1202204]

21. Spitzer, R.; Gibbon, M.; Williams, J. Structured Clinical Interview for Axis I DSM-IV Disorders (SCID). Washington, DC: American Psychiatric Association Press, Inc; 1995.

22. Wills CE, Rovner MH. Preliminary validation of the Satisfaction With Decision scale with depressed primary care patients. Health Expectations. 2003; 6:149-159. [PubMed: 12752743]

23. Hamilton M. A rating scale for depression. Journal of Neurology, Neurosurgery \& Psychiatry. 1960; 23:56-62.

24. Lawton MP, Brody EM. Assessment of older people: self-maintaining and instrumental activities of daily living. The Gerontologist. 1969; 9:179-186. [PubMed: 5349366]

25. Fishman PA, Goodman MJ, Hornbrook MC, et al. Risk adjustment using automated ambulatory pharmacy data: the RxRisk model. Medical Care. 2003; 41:84-99. [PubMed: 12544546]

26. Snaith RP, Baugh SJ, Clayden AD, et al. The Clinical Anxiety Scale: an instrument derived from the Hamilton Anxiety Scale. British Journal of Psychiatry. 1982; 141:518-523. [PubMed: 7150890]

27. Link B, Cullen FT, Struening E, et al. A modified labeling theory approach in the area of mental disorders: an empirical assessment. American Sociological Review. 1989; 54:100-123.

28. Sirey JA, Bruce ML, Alexopoulos GS, et al. Perceived stigma as a predictor of treatment discontinuation in young and older outpatients with depression. American Journal of Psychiatry. 2001; 158:479-481. [PubMed: 11229992]

29. SPSS v14.0. Chicago, Illinois: SPSS Inc.; 2005.

30. Sirey JA, Bruce ML, Alexopoulos GS, et al. Stigma as a barrier to recovery: perceived stigma and patient-rated severity of illness as predictors of antidepressant drug adherence. Psychiatric Services. 2001; 52:1615-1620. [PubMed: 11726752]

31. Sirey JA. The impact of psychosocial factors on experience of illness and mental health service use. American Journal of Geriatric Psychiatry. 2008; 16:703-705. [PubMed: 18757765]

32. Conner KO, Copeland VC, Grote NK, et al. Mental health treatment seeking among older adults with depression: the impact of stigma and race. American Journal of Geriatric Psychiatry. 2010; 18:531-543. [PubMed: 20220602]

33. Fleck DE, Keck PE Jr, Corey KB, et al. Factors associated with medication adherence in African American and white patients with bipolar disorder. Journal of Clinical Psychiatry. 2005; 66:646652. [PubMed: 15889954] 
34. Gerber BS, Cho YI, Arozullah AM, et al. Racial differences in medication adherence: A crosssectional study of Medicare enrollees. The American Journal of Geriatric Pharmacotherapy. 2010; 8:136-145. [PubMed: 20439063]

35. Riedel-Heller SG, Matschinger H, Angermeyer MC. Mental disorders-who and what might help? Help-seeking and treatment preferences of the lay public. Social Psychiatry and Psychiatric Epidemiology. 2005; 40:167-174. [PubMed: 15685409]

36. Sirey, J. Faces of stigma: older adult and provider perspectives. Paper presentation at the annual meeting of the American Association of Geriatric Psychiatry; San Juan, Puerto Rico. 2006.

37. Bao Y, Shao H, Peng T, et al. Diagnosed depression among Medicare home healthcare patients: national estimates of prevalence and key profiles. Psychiatric Services. in press.

38. Loh A, Simon D, Wills CE, et al. The effects of a shared decision-making intervention in primary care of depression: a cluster-randomized controlled trial. Patient Education and Counseling. 2007; 67:324-332. [PubMed: 17509808]

39. Raue PJ, Schulberg HC, Lewis-Fernandez R, et al. Shared decision-making in the primary care treatment of late-life major depression: a needed new intervention? International Journal of Geriatric Psychiatry. 2010; 25:1101-1111. [PubMed: 19946872]

40. Wills, CE.; Franklin, M.; Holmes-Rovner, M. Feasibility and outcomes testing of a patientcentered decision support intervention for depression in people with diabetes. Paper presented at the 4th International Shared Decision Making Conference; Freiburg, Germany. 2007. 


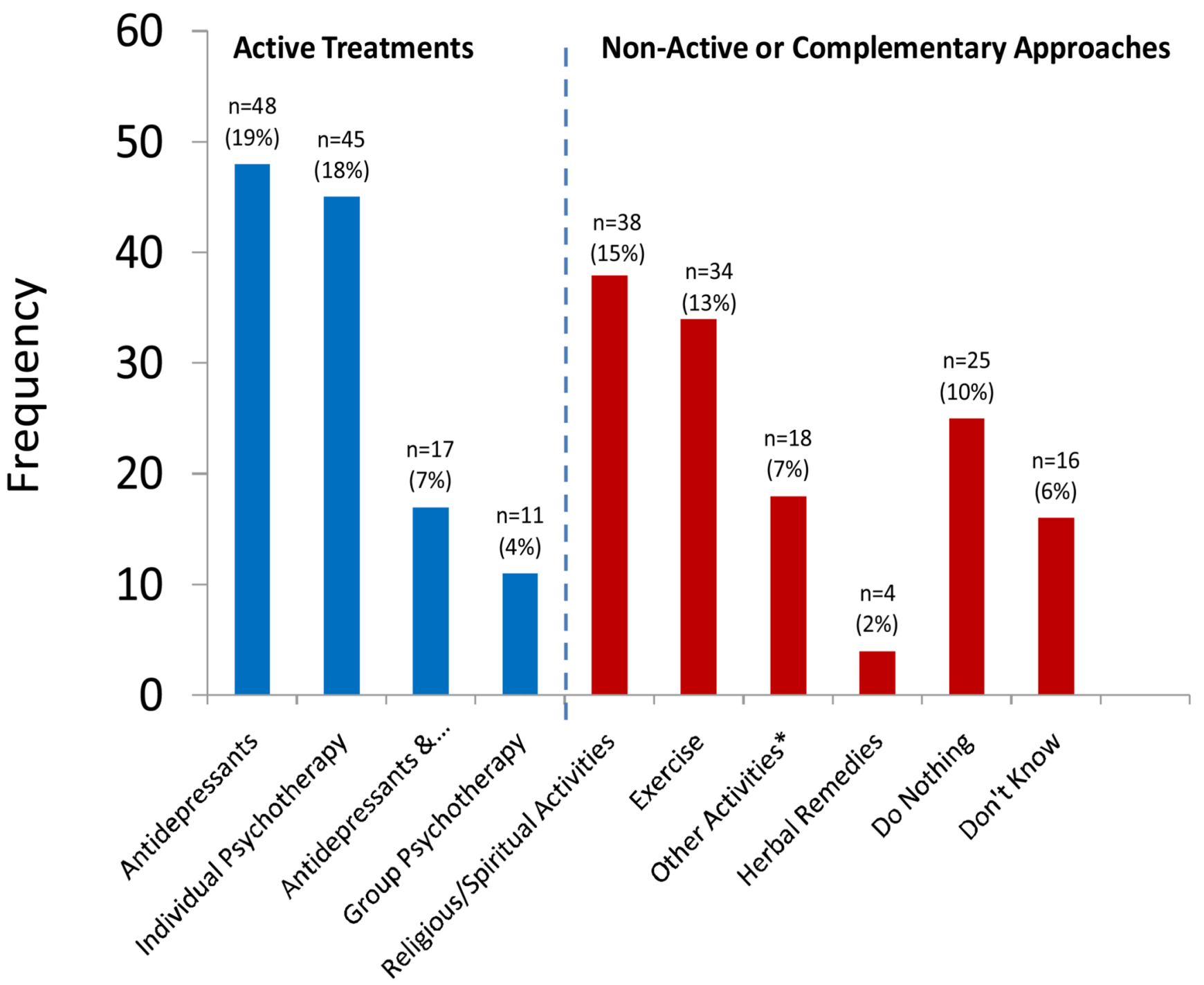

Figure 1. First Choice Treatment Options ( $\mathrm{N}=256)$

*(e.g. talk to family or friends, deal with on own, meditation, music, alcohol use) 


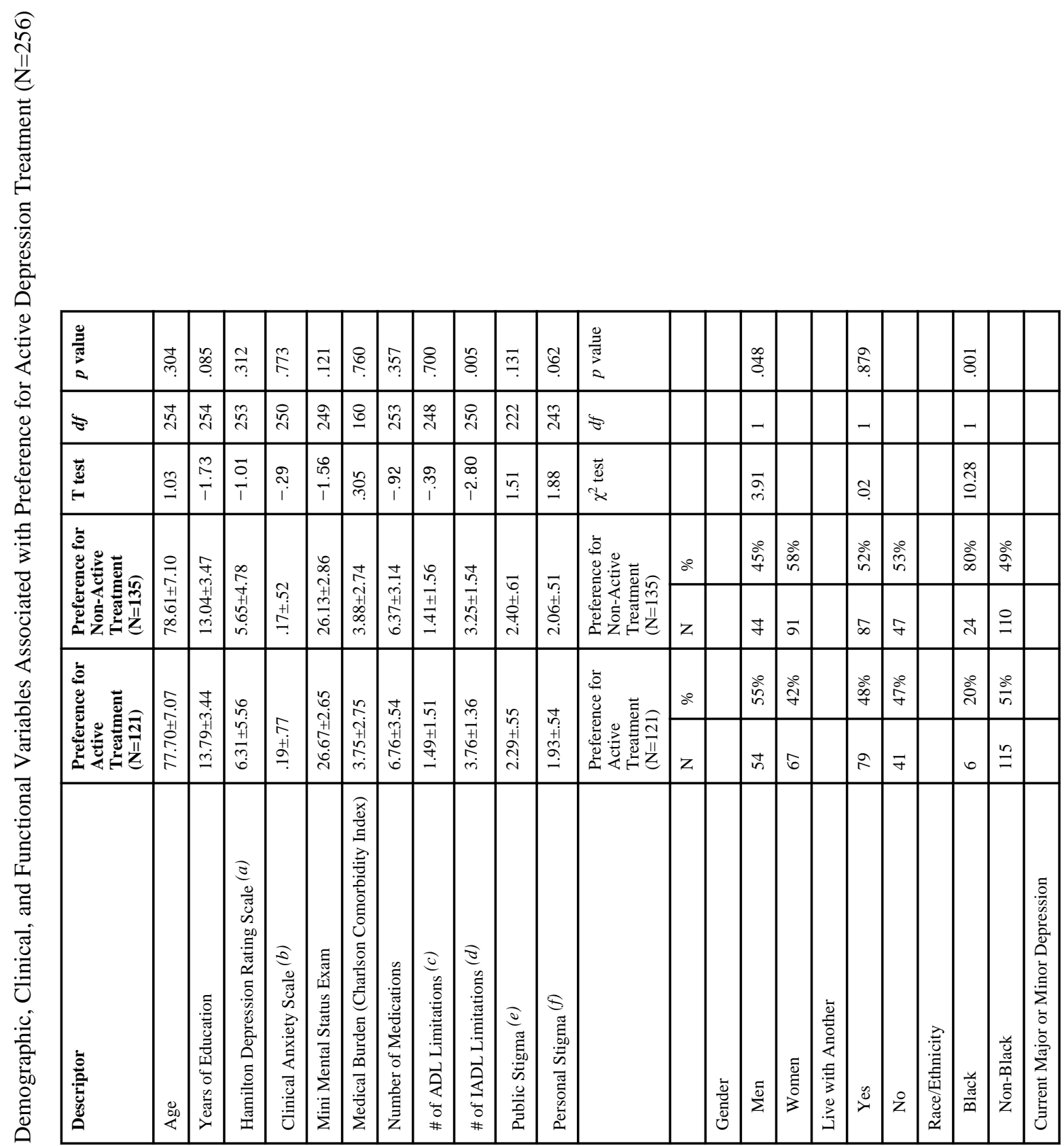




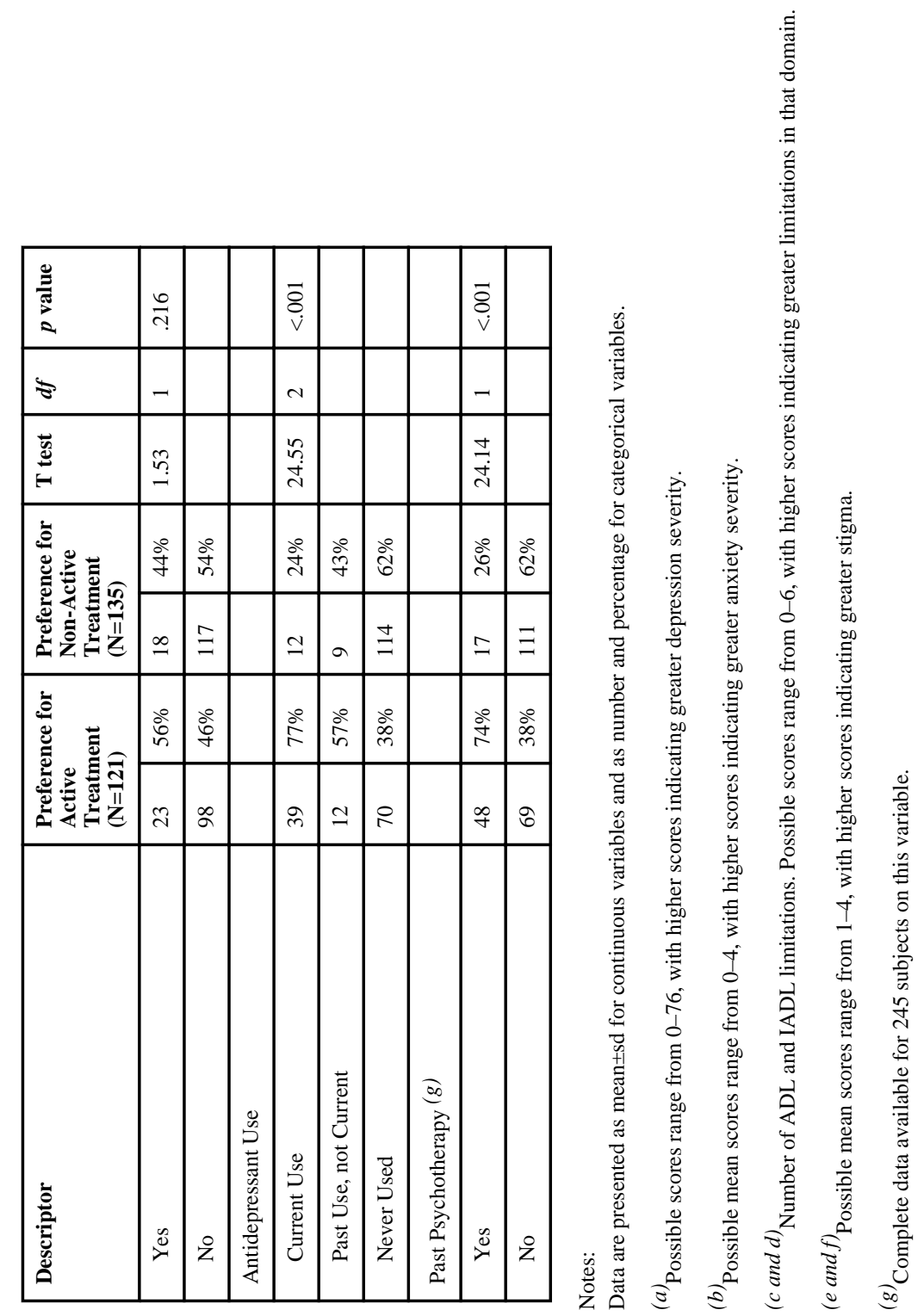

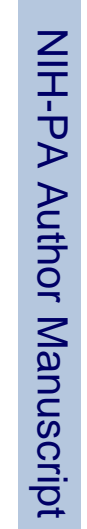




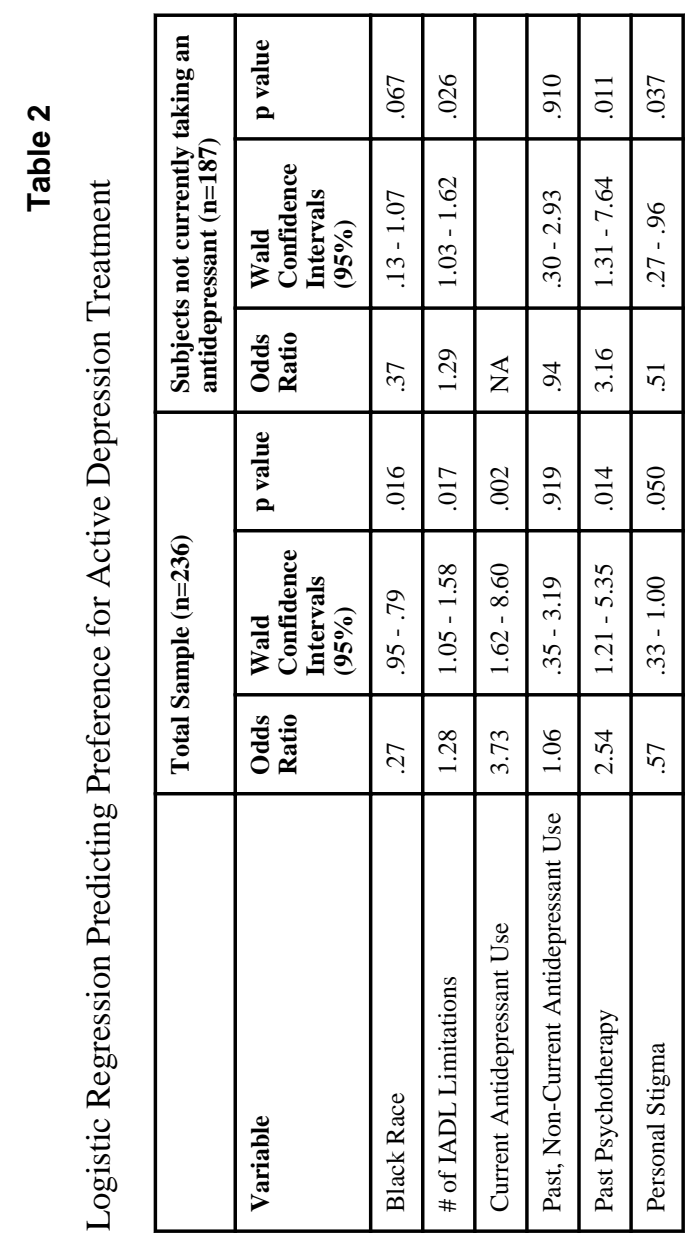

